\title{
Studio preliminare sullo stile parentale percepito in un campione di giovani tossicodipendenti di sesso maschile
}

\author{
Mario G.L. De Rosa, Giuseppina Sanza*
}

- Several factors are considered to underlie the development of dependence. In particular, our study evaluates the relationship between addiction and parental perceptions in a sample of young drug users aged between 1824 years. It is shown that the examinees present a dysfunctional perception of the educational and affective role of their parents with different results between father and mother. The father appears disengaged and negligent, while the mother has a controlling behavior without affection. This condition is associated with the structure in the young of a personality disorder and a specific narcissistic lifestyle. The hypothesis is that the emotional and affective dysregulation of young people surveyed is linked to a problematic parental relationship. The development of dysfunctional personalities and addiction to drugs follow this phenomenological development. The search for drugs aims at modulating and mitigating the emotional and affective dysregulation that these individuals have.

Keywords: parental perception, addiction, personality disorder.

Parole chiave: percezione dei genitori, tossicodipendenza, disturbo di personalità.

\section{Introduzione}

Nel determinismo del comportamento umano non si può considerare esclusivamente e separatamente la dimensione intra-psichica da quella extra-psichica. In particolare, alla base della strutturazione della personalità, la cui fenomenologia si sviluppa in età infantile e adolescenziale, si concepisce una multi-fattorialità causale che comprende aspetti psichici, temperamentali e ambientali, in primis la relazione del soggetto col contesto e il modello di riferimento, soprattutto parentale.

Nella prospettiva della valutazione psicologica, ne deriva che la persona non può essere esaminata in maniera monista: solo individuo o relazione, è rilevante, altresì, una visione olistica, che consideri l'individuo come un Essere nel Mondo che sviluppa una coscienza psichica in funzione dell'interrelazione tra il Sé (inteso come senso onnicomprensivo della dimensione organismica: Corpo-Psiche-Spirito-Relazione) e I'Altro.

Di fatto, lo stile genitoriale risulta condizionante il modo d'Essere del giovane, influenzandone la visione che ha di Sé e del Mondo.

Già Freud (1914) nel testo: "Introduzione al Narcisismo", evidenziava la tendenza alla trasmissione di aspettative narcisistiche dai genitori ai figli.

Questa modalità, nei nostri tempi, è riproposta da Pietropolli Charmet (2008) che descrive, nell'adolescente, la "figura identificativa" di "Narciso" che viene riflessa sia dal contesto parentale sia dal modello esterno-sociale.

* Servizio Dipendenze Patologiche - Dipartimento Dipendenze AsurAV3 di Civitanova Marche.
Oggi il modello genitoriale risulta diverso rispetto al passato, quando era prevalentemente normativo, superegoico, per cui favoriva la strutturazione di "personalità edipiche" declinate, nella condizione di patologia, nella dimensione nevroticodepressiva della colpa, mentre la prevalenza odierna di "personalità narcisiste" caratterizza maggiormente patologie del comportamento.

Date queste premesse, il nostro dato osservazionale, derivante dall'esame clinico e anamnestico di giovani pazienti tossicodipendenti e dei loro genitori, ha evidenziato, preliminarmente, differenze significative e obiettivamente problematiche nei ruoli svolti dal padre e dalla madre nella relazione familiare.

Ne è originato I'interesse di approfondire le caratteristiche di questa dinamica.

In particolare, vista la multi-fattorialità alla base della fenomenologia della Dipendenza, è stato analizzato uno dei fattori ritenuti causali di questa patologia: la relazione familiare e in modo specifico come viene percepito il ruolo dei genitori da parte di un campione di giovani tossicodipendenti afferiti al Servizio.

Diverse ricerche evidenziano dei pattern specifici nella percezione dello stile genitoriale da parte dei soggetti tossicodipendenti. Questi studi hanno preso in considerazione differenti strumenti diagnostici atti alla definizione della funzione auto-percettiva del giovane rispetto alla relazione parentale.

V.C.S. Cavaco e coll. (2009), utilizzando il PBI (Parental Bonding Instrument), ha valutato 100 soggetti di sesso maschile che presentavano una Dipendenza da sostanze stupefacenti, confrontandoli con un gruppo di controllo.

Lo studio ha rilevato, nel campione dei tossicodipendenti, la percezione di un "controllo senza affetto" da parte dei genitori, 
rispetto a una relazione ottimale presente nel gruppo di controllo.

In maniera simile, A. Espina e coll. (1996), applicando il PBI, ha osservato in 60 tossicodipendenti un "minor accudimento" e uno stile di "controllo senza affetto" nella percezione dei genitori, mentre nei 36 soggetti del gruppo di controllo non si evidenziavano problematicità nel rapporto genitore-figlio.

B.R. Garcia (2014), somministrando il PBI ha rilevato, nelle risposte di 100 tossicodipendenti, la percezione di genitori "iperprotettivi e poco accudenti", con un "controllo senza affetto", mentre la FACES III ha evidenziato come questi genitori fossero "distaccati" a livello affettivo, determinandosi un sistema familiare "caotico".

Tali aspetti contrastano con il dato di famiglie "coesive" e "affettive" presente nel gruppo di controllo, costituito da 109 individui di sesso maschile.

In una ricerca su un campione di 846 adolescenti di età compresa tra i 15-20 anni, S.E. Clausen (1996) ha utilizzato una versione "breve" del $\mathrm{PBI}$, riscontrando una correlazione tra consumo di sostanze e percezione genitoriale che è risultata di tipo "controllo senza affetto", con "basso livello di accudimento" e "alto livello di protezione".

Allo stesso modo, R.D. Schweitzer e R.D. Lawton (2006), applicando il PBI su un campione di 63 tossicodipendenti maschi e femmine, hanno individuato una percezione della relazione parentale di tipo "fredda", "indifferente", "controllante" e "intrusiva".

Tra gli studi italiani è da segnalare quello di C. Agostino e coll. ((2008).

Nel corso della ricerca è stata somministrata la FACES III a 24 nuclei familiari in cui era presente un figlio tossicodipendente.

L'autore ha evidenziato la percezione di "genitori distanti e rigidi", con I'area della "flessibilità" critica rispetto a quella della "coesione".

Nell'ambito degli studi clinici ricordiamo, infine, la ricerca di Cirillo e coll. (1996).

Questi, analizzando un campione di circa 200 soggetti di sesso maschile e le loro famiglie, ha verificato che la "storia ricorrente" dei tossicodipendenti maschi si differenzia da quella delle ragazze tossicodipendenti, soprattutto per la trasmissione di "carenze di accudimento" genitoriale, con una modalità transgenerazionale.

Cirillo ha osservato una disfunzionalità relazionale in entrambi i genitori del soggetto tossicodipendente, riferendola a un disagio sperimentato nella relazione problematica con i rispettivi genitori. La deficitarietà vissuta nell'infanzia e nell'adolescenza non viene da loro riconosciuta in quanto in essi operano meccanismi di minimizzazione e di misconoscimento del "trauma" subito: il padre spesso è stato adultizzato precocemente per l'"assenza" del genitore e col figlio, non riconoscendo il suo stigma, ripete un ruolo "periferico" in maniera inconsapevole, delegando alla moglie il compito di ordinatrice e latrice delle regole rispetto all'educazione del giovane.

La madre del tossicodipendente, a sua volta, ha vissuto un invischiamento relazionale con la propria madre, derivandone una coartazione affettiva, per cui divenuta adulta abdica dal ruolo di accudimento del figlio, affidandolo spesso alla sua stessa genitrice.

L'accudimento difettuale si riflette nella disposizione del giovane a sviluppare problematicità comportamentali, correlate, in particolare, al consumo di sostanze stupefacenti.

In questa prospettiva, risulta significativa la riflessione di Pietropolli Charmet (1990) che rileva, alla base della tossicodipen- denza, una maternalizzazione della relazione parentale: il padre non svolge un ruolo ordinativo come dovrebbe, demandando alla moglie la definizione delle norme educative del figlio.

Perciò, il giovane, soprattutto in età adolescenziale, vive una crisi esistenziale che origina dall'impossibilità di strutturare un progetto personale per il futuro che gli appare incerto e indefinito, essendo assorbito totalmente nell'area materna.

\section{Soggetti e metodi}

Sono stati esaminati n. 12 soggetti di sesso maschile, di età compresa tra i 18-24 anni, con diagnosi di "Dipendenza da oppiacei" definita secondo i criteri diagnostici del DSM IV-TR e di "Disturbo da uso di oppiacei" in osservanza dei criteri diagnostici del DSM 5.

Il campione, esclusivamente di sesso maschile, è stato così individuato per rendere omogenei i risultati del vissuto percepito rispetto allo stile genitoriale.

La valutazione del campione è stata effettuata utilizzando i seguenti strumenti psicodiagnostici:

1. SCL 90 (Symptom Checklist-90), di L. Derogatis (1973): è un questionario di 90 item che riflette la configurazione di sintomi psicologici di soggetti non clinici e clinici, la SCL-90 valuta nove dimensioni sintomatologiche primarie: somatizzazione, ossessività-compulsività, ipersensibilità interpersonale, depressione, ansia, ostilità, ansia fobica, ideazione paranoide, psicoticismo.

2. PBI (Parental Bonding Instrument), di G. Parker et al. (1979). È un questionario auto-compilato che misura la percezione dei comportamenti ricevuti dal giovane nel corso dell'infanzia da parte dei genitori. Le informazioni devono basarsi sul ricordo che il paziente ha dei rapporti sperimentati con i genitori nei primi 16 anni di vita. Il questionario è costituito da 25 item: 12 item esplorano la dimensione delle cure parentali, mentre 13 item la dimensione dell'iperprotezione/controllo.

3. FACES III (Family Adaptation and Cohesion Scales), di D.H. Olson (1985). È un questionario costituito da 2 fogli contenenti 20 item ciascuno. Nel primo foglio il paziente descrive com'è la propria famiglia a livello reale, nel secondo foglio come vorrebbe che fosse (livello ideale).

La discrepanza tra il livello reale e quello ideale permette di avere informazioni sul grado di soddisfazione del soggetto in rapporto alla famiglia. Dal questionario si evincono diverse tipologie di funzionamento familiare, prendendo in considerazione due dimensioni: la Coesione e la Flessibilità. Per Coesione s'intende il legame emotivo tra i membri di una famiglia, per Flessibilità la capacità del sistema di cambiamento di regole, ruoli e funzioni.

Il funzionamento ottimale è caratterizzato da confini flessibili e comunque definiti sia all'esterno sia all'interno, da un'elasticità dei ruoli, da una leadership condivisa e da una struttura sistemica tale da favorire un equilibrio tra appartenenza e separazione. Questo funzionamento lo ritroviamo nell'area centrale, considerata dall'autore I'Area Bilanciata, che comprende, nella dimensione della Coesione, un funzionamento definito "separato-connesso" e nella dimensione della Flessibilità, un funzionamento "strutturato-flessibile", mentre agli estremi troviamo sistemi rigidi, caotici, invischiati e disimpegnati.

In particolare, i livelli di coesione molto alti (invischiamento) e i livelli di coesione molto bassi (disimpegnato) possono risultare problematici per i membri della famiglia; lo stesso 
vale per la dimensione della Flessibilità i cui livelli molto alti (caotici) e i livelli molto bassi (rigidi) possono risultare, a lungo andare, disfunzionali; il funzionamento familiare che si situa nell'area centrale/bilanciata, caratterizzata da relazioni strutturate e flessibili, promuove, altresì, in modo funzionale, un equilibrio tra i cambiamenti e la stabilità.

4. Il vissuto percepito dai soggetti è stato valutato, inoltre, per mezzo dell'Analisi del craving (M.G.L. De Rosa, 2005), una tecnica psicologica originale che prevede l'elicitazione emozionale e del desiderio precedente al craving, col fine di comprendere il "senso" della dipendenza della persona, essendo il craving l'epifenomeno equivalente della dipendenza psicologica.

\section{Risultati}

Rispetto all'uso di sostanze stupefacenti n. 8 soggetti usano eroina come sostanza primaria, n. 2 cannabinoidi, n. 2 cocaina. Da un punto di vista psichico tutti i giovani esaminati presentano una personalità narcisistico-borderline. Tale valutazione è derivata dall'elaborazione della testistica psicodiagnostica (SCL 90), dall'applicazione dei criteri diagnostici del DSM-IV TR e del DSM 5 integrati con un inquadramento personologico secondo I'organizzazione psicopatologica proposta da Otto Kernberg (1975).

Rispetto alla relazione parentale, elaborando il PBI si evidenzia, nel campione, la percezione di "genitori disimpegnati", con una scarsa "vicinanza" ai figli e una spinta alle singole autonomie. La figura materna è prevalentemente "controllante senza affetto", più "normativa-controllante" della figura paterna che appare "negligente/assente", "non normativa" e non risulta essere neppure affettiva. Relativamente alle risposte del PBI rispetto alla percezione della madre, n. 7 soggetti hanno una percezione di tipo "controllante-senza affetto", n. 4 di "genitore ottimale", n. 1 di "genitore negligente".

Riguardo alla figura paterna: n. 7 soggetti percepiscono un "genitore negligente", n. 2 soggetti un "genitore controllante senza affetto" e solo n. 3 soggetti un "genitore ottimale".

La FACES III ha individuato, nel campione esaminato, un ideale parentale di genitore normativo, affettivo, non controllante, mentre nella percezione della famiglia "reale", n. 8 soggetti hanno descritto il nucleo parentale come "rigidamente disimpegnato", n. 3 come "flessibilmente separato", n. 1 come "strutturalmente separato".

La diagnosi del quadro psicopatologico e della Dipendenza si è avvalsa anche dell'Analisi del craving che ha permesso di correlare la condizione di Dipendenza con la problematica psicopatologica del soggetto (M.G.L. De Rosa, 2013).

In particolare, dall'analisi dei vissuti relati al craving, è emersa la frustrazione del desiderio di "affermazione di sé" che caratterizza il costrutto identitario di questi soggetti. Si è rilevata la stretta connessione tra la disconferma di sé e la percezione di una relazione parentale disarmonica.

Il senso della mancanza, nell'ottica di un rispecchiamento emotivo-affettivo inadeguato con i genitori, si associa a un modo d'essere disregolato, a una personalità difettuale nella dimensione emotivo-affettiva, derivandone una coscienza di sé dereistica e ipertrofica, fragile e poco definita.

L'ideale di sé di questi soggetti, deprivati a livello affettivo e nelI'auto-percezione emotiva, è orientato su istanze pulsionali edonistiche, alla ricerca di un piacere nel versante sensoriale, essendo carenti nella capacità di esperire un piacere affettivo.
Dal vissuto percepito, risulta la figura di un padre "fuori ruolo educazionale" che esprime un modo d'essere infantile al pari del figlio, mentre la madre vive un ruolo frustrato come moglie, con una modalità di relazione familiare di tipo ambivalente, instabile da un punto di vista emotivo-affettivo.

La frustrazione che prova verso il marito riguarda il desiderio di "essere riconosciuta come donna da amare", mentre come madre avverte la disconferma del desiderio di: "essere amata dal figlio" che di fatto, gradualmente, si separa da lei. Ciò implica nella donna un'ipercompensazione narcisistica che si riflette in una relazione simbiotica con il figlio, condizione che ipertrofizza ulteriormente la sua dimensione personologica narcisistica. Rispetto al marito, il sentirsi rifiutata nel desiderio, le induce, altresì, sentimenti di rabbia, rancore e rivendicazione.

Questi aspetti contrastano con il ruolo genitoriale fisiologico: il padre nella dinamica familiare dovrebbe risultare "ordinativo", un modello razionale che trasmette al figlio la "dimensione del Senso", guidandolo nell'esame di realtà e nell'adattamento alle regole e alle leggi sociali, mentre la madre dovrebbe svolgere il ruolo del caregiver che rispecchia nel giovane una maturazione affettiva.

Di fatto, il padre tende a colludere col figlio nelle richieste narcisistiche, la madre risulta, altresì, disorientata, arrabbiata, ipoaffettiva: entrambi evadono, in questo modo, dal loro ruolo educativo.

\section{Conclusioni}

L'originalità della nostra ricerca si basa su tre aspetti essenziali: 1. si è preso in esame un campione di tossicodipendenti di sesso maschile con un range di età giovanile, 18-24 anni, rispetto al dato della letteratura esaminata che prevede studi, generalmente, senza definizioni di pattern specifici di età.

Questo significa che la percezione dello stile genitoriale, essendo il campione costituito da giovani, non deriva da un ricordo adolescenziale, ma da un vissuto recente che può considerarsi autentico, non contaminato da ulteriori esperienze avvenute nel corso della vita adulta.

Inoltre, I'analisi di un campione omogeneo per sesso, ha permesso di elicitare $\mathrm{i}$ vissuti dei soggetti esaminati in maniera uniforme, evitando confrontazioni di genere potenzialmente confusive.

2. Relativamente alla percezione dello stile genitoriale sono stati utilizzati due questionari: il PBI e la FACES III.

Ciò ha facilitato I'individuazione della percezione dei soggetti esaminati, grazie anche alla comparazione dei dati ottenuti nei singoli test.

Nella letteratura specifica, di norma, è stato utilizzato solo un questionario.

3. I dati percettivi sono stati valutati anche come vissuti individuali e analizzati tramite una tecnica psicologica originale: I'Analisi del craving che ha permesso di evidenziare ed elaborare il senso del vissuto emozionale percepito dal giovane. In questa prospettiva si è potuto definire, nel soggetto, l'ideale personale frustrato in relazione al contesto familiare, correlando tale vissuto con la strutturazione del modo d'essere patologico che il giovane manifesta, elicitandone il significato esistenziale e l'evoluzione tossicomanica.

Riguardo al fenomeno della Dipendenza, analizzando i dati anamnestici e il vissuto che emerge dall'Analisi del craving, è stato rilevato, in tutti i soggetti esaminati, I'iniziazione e lo sviluppo del consumo di sostanze stupefacenti secondo la seguen- 
te modalità: le prime esperienze tossicofile sono avvenute in un'età compresa tra i 13-14 anni, caratterizzate da un uso sporadico di cannabis.

Il consumo è diventato giornaliero nel corso di 1-2 anni. Intorno ai 15-16 anni, il giovane ha sperimentato la cocaina e droghe anfetaminiche, quindi l'eroina "fumata", prima saltuariamente poi in modo continuativo.

In merito ai vissuti, risultano specifici e omogenei rispetto alla percezione del ruolo svolto dal padre e dalla madre nella dinamica familiare, evidenziando una modalità relazionale problematica.

Nella dinamica familiare il padre dovrebbe risultare "ordinativorazionale", autorevole figura-guida del figlio col compito di favorirne l'acquisizione di un Senso personale rispetto al mondo esterno e alle sue regole e leggi, mentre la madre dovrebbe rappresentare il rispecchiamento della dimensione affettiva, finalizzata alla strutturazione di un sentire interiore nel figlio.

La discrepanza tra il ruolo atteso dai genitori e quello percepito dai giovani tossicodipendenti conferma il dato osservazionale di uno stile e una configurazione parentale centrata soprattutto su istanze narcisistico-onnipotenti.

Si è rilevato, inoltre, che la problematicità dei genitori, in base ai dati anamnestici in nostro possesso, segue una modalità di trasmissione transgenerazionale.

In particolare, il padre tende a colludere col figlio nelle richieste narcisistiche, al contrario la madre rimane disorientata e pervasa da un sentimento di rabbia, una volta che esperisce nella relazione con il marito e con il figlio una disconferma rispetto al suo desiderio affettivo che appare ipertrofico, simbiotico, dereistico, mosso da un "volere" onnipotente.

Nel corso della crescita fisiologica, I'adolescente dovrebbe bilanciare da un lato la relazione parentale per separarsi e al contempo sperimentare la sua capacità relazionale, cognitiva (Sé intellettuale) e corporea (Sé corporeo).

Questo processo comporta una ristrutturazione del Sé e una valutazione del proprio senso di efficacia, operazione molto delicata.

Perciò se si verifica una rigidità intra-familiare come quella descritta e se vive al contempo stressors esterno-ambientali, può esperire un "break-down" che gli determina una profonda e intensa frattura interiore.

La discrepanza esistenziale verte soprattutto tra l'ideale che il giovane ha del genitore: maggiormente normativo, affettivo e non controllante, rispetto al dato realistico che viene vissuto in maniera problematica.

Di fatto, la dimensione narcisistica genitoriale riflette la prevalente dimensione narcisistica dell'odierno modello sociale, per cui il giovane, pur aspirando a un'educazione armonica, deve confrontarsi spesso con pattern di riferimento e identificativi disfunzionali e pervasivi sia intra che extra-familiari.

Questa condizione può determinare una regressione pulsionale e una disregolazione emotivo-affettiva che non contempla una progettualità esistenziale definita ed equilibrata nel mondo, ma un progetto proiettato sull'immediato.

La fenomenologia esistenziale descritta risulta ulteriormente destrutturante quando il giovane presenta un temperamento condizionato al "reward" e/o al "sensation seeking", associato a un'ipersensibilità emotiva costituzionale o comunque derivata da una Relazione d'attaccamento disfunzionale (ambivalente, evitante o disorganizzata).

Tale condizione psico-relazionale, se particolarmente intensa e deficitaria nelle life-skills e nella capacità di coping, può orientare il giovane a sperimentare sostanze stupefacenti col fine auto-terapeutico di placare lo stato di angoscia e di smarrimento che prova.

Da queste osservazioni che descrivono una potenzialità evolutiva problematica, in cui l'indefinitezza personologica si associa a comportamenti incongrui e compulsivi, relata a un rispecchiamento emotivo-affettivo disfunzionale col genitore, deriva l'importanza di strutturare una modalità di relazione educativa che sia quanto più fondata su codici formativi solidi, chiari, orientati a definire un'identità autentica e armonica, in rapporto, soprattutto, col mondo esterno.

In questa prospettiva, come intervento di prevenzione primaria, si considera congrua la possibilità che i genitori usufruiscano, se motivati, di Centri di ascolto dove operino figure specialistiche in grado di fornire loro risposte adeguate e professionali rispetto a domande di tipo educativo.

Riguardo al quadro personologico associato alla condizione di Dipendenza che è stato rilevato essere essenzialmente di tipo "narcisistico-borderline", la letteratura scientifica conferma la prevalenza di questa strutturazione psichica, evidenziando, di fatto, nella popolazione dei tossicodipendenti, frequenti disturbi di personalità caratterizzati da una maggiore difficoltà nel controllo degli impulsi, da una disposizione agli agiti autolesivi e al comportamento antisociale, riconducibili, in particolare, al Disturbo Borderline, al Disturbo Narcisistico e al Disturbo Antisociale di Personalità (Gabbard, 2007).

Tali quadri psicopatologici si sviluppano da vissuti infantili di trascuratezza, dalla mancanza di una "vicinanza amorevole" del caregiver, fino al maltrattamento/abuso (Benjamin, 1996).

Nel corso di questi sviluppi problematici, come rilevano Cancrini (2012) e Colacicco (2013), i giovani possono risultare a rischio di evoluzioni tossicomaniche.

Nello specifico della nostra ricerca, la condizione psicopatologica e la strutturazione della Dipendenza da sostanze stupefacenti evidenzia una correlazione significativa tra questi disturbi e una storia relazionale, soprattutto familiare, nella quale il rapporto del figlio con i genitori è risultato disfunzionale: non si è definito un accudimento parentale adeguato e armonico, prevalendo relazioni distanzianti, svalutanti, ipo-anaffettive e controllanti.

In conclusione, il nostro studio, di tipo preliminare, visto il campione ridotto di soggetti esaminato, seppure limitato in termini quantitativi, rileva un dato qualitativo suggestivo: la presenza, nei soggetti esaminati, di un'associazione tra Dipendenza da oppiacei, Disturbi di Personalità del Cluster B (DSM 5) e la percezione problematica del caregiver: padre e madre.

Il dato clinico-osservazionale, derivato dall'analisi del vissuto dei giovani pazienti, da cui si evince che la dimensione psicorelazionale dei genitori presenta primariamente tratti di tipo narcisistico-borderline, sottolinea la trasmissione trans-generazionale della problematica psichica.

Al contempo, si evidenzia il rischio che tali strutturazioni personologiche, carenti e disregolate a livello emotivo-affettivo, determinino nel giovane modalità comportamentali disfunzionali alla ricerca di relazioni oggettuali in grado di colmare e "regolare" il vuoto e la frustrazione affettiva ed emotiva che prova, che risulta associata a una mal-strutturazione personologica derivante da una Relazione d'attaccamento vissuta come problematica e insoddisfacente.

Il craving verso le sostanze psicoattive e stupefacenti può essere interpretato in quest'ottica fenomenologica e psicopatologica, anche se nel determinismo dei percorsi di Dipendenza risultano comunque significative le predisposizioni temperamentali e i fattori extra-familiari. 
In particolare, nella definizione dello stile parentale agiscono anche i modelli di riferimento sociali: quelli odierni enfatizzano, soprattutto, la dimensione individuale narcisistica e borderline $\mathrm{e}$ favoriscono, di conseguenza, la strutturazione di un modo d'essere disfunzionale, centrato, in maniera autoreferenziale su un Sé Grandioso.

Lo stile personale non prevede quindi solo determinanti endopsichiche: il "pressing" esterno, se particolarmente intenso, è in grado di condizionare, già a livello infantile, i comportamenti dell'individuo.

Per questo motivo, riteniamo che gli obiettivi clinici e di ricerca applicati a queste problematiche debbano considerare non solo la modalità relazionale di tipo duale: caregiver-figlio, ma anche la dinamica che avviene all'interno della triade: caregiver-figliomodello sociale.

\section{Bibliografia}

1. Agostino C., Bertolotti M.C., Porcella E., Visani E. (2008), "Tossicodipendenza e relazioni familiari: uno studio con FACES III", Rivista di Psicoterapia Relazionale, n. 28: 73-90.

2. Benjamin L.S. (1996), Interpersonal Diagnosis and Treatment of Personality Disorders, The Guilford Press, New York, trad. it. Diagnosi interpersonale e trattamento dei disturbi di personalità, LAS, Roma, 1999, pp. 141-199.

3. Cancrini L. (2012), La cura delle infanzie infelici, Raffaello Cortina, Milano, p. 26.

4. Colacicco F. (2013), La mappa del terapeuta, Scione, Roma, p. 79

5. Cavaco V.C.S., Neves de Jesus S., de Rezende M.M. (2009), "Percepcao de estilos parentais na toxodependencia", Boletin de Psicologia, vol. 59, n. 131, Sao Paulo.

6. Cirillo S., Berrini R., Cambiaso G., Mazza R. (1996), La famiglia del tossicodipendente, Raffaello Cortina, Milano, pp. 40-52.
7. Clausen S.E. (1996), "Parenting styles and adolescent drug use behaviours", Childhood, August, vol. 3, n. 3: 403-414.

8. Derogatis L.R., Lipman R.S, Covi L. (1973), "SCL-90: an outpatient psychiatric rating scale-preliminary report", Psychopharmacol. Bull., Jan., 9(1): 13-28.

9. De Rosa M.G.L., Pierini C., Grimaldi C. (2005), Alcolismo: analisi del craving, FrancoAngeli, Milano.

10. De Rosa M.G.L. (2013), Analisi fenomenologica della Dipendenza. Diagnosi e Psicoterapia, Aracne, Roma, pp. 165-184.

11. Espina A., Ayerbe A., Pumar B., Garcia E. (1996), "La percepcion de las pautas parentales de crianza en toxicomanos", Addicciones, vol. 8, n. 2: 203-217.

12. Freud S. (1914), "Introduzione al narcisismo", OSF, vol. 7: 460461.

13. Gabbard G.O. (2007), Psychodinamic Psychiatry in Clinical Practice. Fourth Edition, American Psychiatric Press, Inc., trad. it. Psichiatria psicodinamica, Raffaello Cortina, Milano, pp. 359-365.

14. Garcia B.R. (2014), "Percepcion de los vinculos parentales y funcionamento familiar en sujetos drogodependientes. Un recurso a explorar en el proceso de rehabilitacion", Revista PsiqueMag, Octubre, vol. 3, n. 1.

15. Kernberg O. (1975), Borderline conditions and pathological Narcisissism, Jason Aronson, New York, trad. it. Sindromi marginali e Narcisismo patologico, Bollati Boringhieri, Torino, 1978, pp. 2745.

16. Olson D.H., Portner J., Lavee Y. (1985), FACES III (Family Adaptation and Cohesion Scales), University of Minnesota. St. Paul, MN.

17. Parker G., Tupling H., Brown L.B. (1979), "A parental bonding instrument", British Journal of Medical Psychology, 52: 1-10.

18. Pietropollli Charmet G. (1990), L'adolescente nella società senza padri, Unicopli, Milano, pp. 38-46.

19. Pietropolli Charmet G. (2008), Fragile e spavaldo. Ritratto dell'adolescente di oggi, Laterza, Bari, pp. 3-34.

20. Schweitzer R.D., Lawton P.A. (1989), "Drug abusers' perceptions of their parents", British Journal of Addiction, vol. 84, n. 3: 309-314. 